\title{
Effect of cu and co nanoparticles based complex on stratification of grape grafts
}

\begin{abstract}
The aim of the given work was to investigate the influence of copper and cobalt nanoparticles on clonal micro reproduction of Johanniter grape technical variety received by method of isolated cells and tissues culture. Copper and cobalt nanoparticles were used in the form of colloidal solutions of high-viscosity (gels). Johanniter grape technical variety was used as an object of research. During experiments the optimum concentrations of copper $(0.5,1.0,1.5 \mathrm{mg} / 1)$ and cobalt $(0.5,1.0,1.5 \mathrm{mg} / \mathrm{l})$ nanoparticles in colloidal solutions have been defined. These solutions were added in Murasige and Skoog nutrient medium. It is shown that $1 \mathrm{mg} / 1$ concentration of cobalt nanoparticle provides the highest coefficient of reproduction $(1: 17)$. The similar concentration of copper nanoparticles in the colloidal solution has not demonstrated a set positive dynamics.
\end{abstract}

Keywords: simultaneous electron beam evaporation and deposition, co, cu nanosized powders, zeta potential, particle size, colloidal solution, clonal micro reproduction, johanniter grape technical variety, in vitro, chromatography, cultivation, growth index, quantity of internodes, quantity of shoots, reproduction coefficient
Volume 4 Issue 2 - 2018

\author{
Shkopinskij EA,' Talankova-Sereda TE, ${ }^{2}$ \\ Liapina KV, ${ }^{3}$ Dulnev PG ${ }^{4}$ \\ 'Zaporozhian National University, Ukraine \\ ${ }^{2}$ National University of Life and Environmental Sciences of \\ Ukraine, Ukraine \\ ${ }^{3}$ EO Paton Electric Welding Institute, Ukraine \\ ${ }^{4}$ Institute of Bioorganic Chemistry and Petrochemistry, Ukraine
}

Correspondence: Liapina KV, EO Paton Electric Welding Institute, NASU, I I K Malevicha str, Kyiv-03 I50, Ukraine, Email kirlyap8I@gmail.com

Received: February 12, 2018 | Published: March 01, 2018

\section{Introduction}

South and South-Eastern regions of Ukraine have unique climatic conditions for wine-making. The latter today is represented by single farmers, therefore, its expansion requires application of modern biotechnological methods, namely method of isolated cells, tissues and organs culture in vitro, micro reproduction for growing the planting material free of bacterial cancer and other diseases. ${ }^{1}$ It has well known that the technical varieties of grapes have a number of problems. One of them is a tendency of this crop to bacterial, fungal and virus diseases, which significantly reduce capacity and longevity of plantings. An infection can take place through the soil as well as the grapevine, then the disease is transmitted during grafting, since a graft can contain a pathogen and be dangerous for the whole future planting. ${ }^{2}$ Thus, it is necessary to get a sufficient number of recovered in vitro microclone cultures and adapt them to in vivo conditions. ${ }^{3,4}$ Johanniter grape technical variety was used as an object of investigation. It should be noted that the Johanniter variety is a complex interspecies hybrid of German breeding, resistant to mildew, oidium and having high winter resistance. It is known fact that microelements play an important role in the regulation of growth processes, including in the in vitro cultures, since they are precursors and stimulators of synthesis and operation of many enzymes. Thus, copper through enzymes effects carbon and nitrogen metabolism, increases plants' resistance due to effect on growth inhibitors of phenolic nature. Presence of nanopowders of copper of 2,0-2,5 nm size in a nutrient medium when coaching the wheat seeds and vetch in a concentration range of $0,01-1,0 \mathrm{~g}$ per a hectare seeding rate provoked a significant increase in germinating energy and similarity. ${ }^{5}$ Cobalt is contained in plants in ionic form and as a constituent of vitamin B12, activates phosphoglyucomatase, arginase and nitrogenase, enolases and kinases, participates in oxidation processes and synthesis of leggemoglobin. The nanoparticles have an extremely high activity and their size corresponds to the size of living cells, therefore, biogenic metals in nanoparticle form are more efficient and safe perception by plants, and the doses necessary to put them into the nutrient medium are significantly smaller. According to references, 1-10 mg/ $1^{6}$ is a physiologically grounded concentration of working solution of biogenic metals nanoparticles. Our previous experiments proved positive influence of cobalt and copper nanoparticles on horsemint (M. longifolia). ${ }^{7,8}$ Therefore, investigation of effect of the biogenic metals nanoparticles on growth characteristics of grape explants, depending on their concentration, is relevant. Thus, the purpose of our research is to study the processes of morphogenesis in vitro in the Johanniter grape technical variety in cultivation of the explants on modified Murasige and Skoog nutrient medium with different content of copper and cobalt nanoparticles.

\section{Methods}

In the first stage, production of pure copper and cobalt in nanoform was based on an approach related with the synthesis of nanoparticles in a chemically inert matrix, which protects them from oxidation and isolates one from another. This ensures display of their individual and very often unique properties. This method, described in details in,, 10 lies in proving an encapsulation of the particles into a chemically inert medium. Process of formation of the copper and cobalt particles from vapor phase was carried out in the presence of vapors of alkali metals halogenides. Presence of a shell on surface of the particles prevents their consolidation during storage and protects it from interaction with the atmosphere. This allows long-term storage of such particles in standard atmosphere, and, if necessary, removes the shell by placing these powders, for example, into a liquid, in which this salt dissolves. In the second stage, in order to obtain a regulator growth based on nanoparticles in form of a high-viscosity colloidal solution, the powder was dispersed in PEGs composition (PEG 1500-8000 in 
4-7 parts volume and PEG 400-600 in 3-6 parts volume). This method is described in details in. ${ }^{11}$ This approach allows obtaining a highviscosity colloidal solution (gel) that can be stored in a compact form for a long time. The properties of received colloidal solutions were investigated by means of Malvern Zetasizer Nano S device (Malvern Instruments Ltd., the Great Britain) Ver. 6.20, in which particles size is defined on their moving with use of Einstein-Stokes relation. Distribution of particles on sizes in colloidal solutions is resulted on Figure 1 (for copper and for cobalt). Distribution is monomodal with a mode and satisfactorily obey the lognormal distribution, which lays in the range of sizes from 0.5 to $2.0 \mathrm{~nm}$.

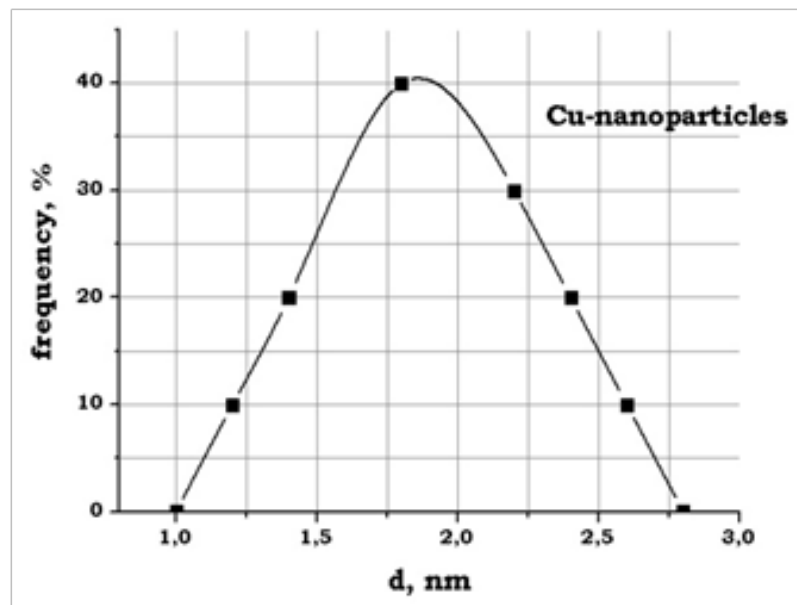

(a)

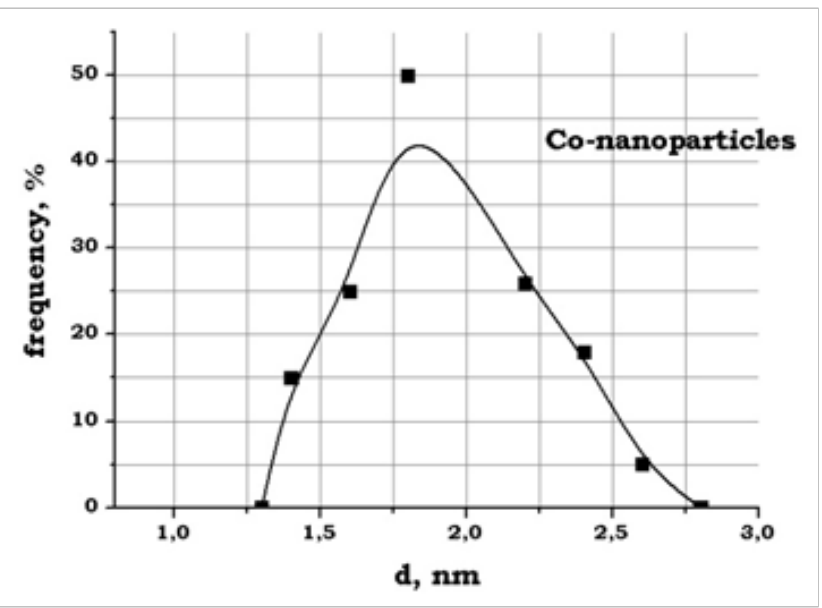

(b)

Figure I Distribution of copper and cobalt particles on sizes in colloidal solutions based on amines.

Further studies were carried out in 2017 in the laboratory of biotechnology in Zaporozhye. Johanniter grape technical variety from the State Institute of Wine-making, Freiburg, Germany has been used as an object of research. 90 explants with $\mathrm{Cu}$ and 90 explants with $\mathrm{Co}$ were used for investigation and all experiments was repeated three times. The explants were injected into the in vitro culture three times, from February to May. Apical and lateral meristems were used for injection by 30 pcs of each kind of plants. Steady-state sterilization was used to obtain sterile material. For this, the vine was cut into segments of 2-4 cm with one node, washed under running water for 20 minutes and then immersed in a solution of Twin-20 for 10 min. Further sterilization was carried out in a laminar box. The explants were placed in a $70 \%$ solution of ethyl alcohol for 30 seconds, after that they were immersed by 6 minutes in a $0.1 \%$ solution of sublimate, and then they were kept for 5 minutes. In $0,05 \%$ solution of $\mathrm{AgNO}_{3}$. The explants were washed from the residues of sterilizing agents with sterile distilled water five times for 15 minutes. The sterile explants were taken using a set of sterile instruments under aseptic conditions according to standard methods ${ }^{12}$ and cultured in nutrient medium of the MS without growth regulators for 30 days in order to ensure absence of contamination. For the 30th day of cultivation in MS medium without growth regulators, the viable explants having no signs of contamination were grafted to 5-7 mm segments with one node, passaged in new MS nutrient media enriched with growth regulators $1.0 \mathrm{mg} / 1$ 6-benzylaminopurine (6-BAP), $0.2 \mathrm{mg} / \mathrm{L}$ adenine,
$0.1 \mathrm{mg} / \mathrm{L}$ indolyl-3-acetic acid (IAA) and $0.5 \mathrm{mg} / 1$ gibberellic acid (GA) as well as copper and cobalt nanoparticles at $0.5 ; 1.0$ and 1.5 $\mathrm{mg} / \mathrm{l}$ concentrations. Control was carried out in the nutrient medium of similar composition without nanoparticles. Ascorbic acid was added to the nutrient medium in $25.0 \mathrm{mg} / \mathrm{l}$ concentration to suppress action of phenolic compounds. The nutrient medium was sterilized in an autoclave under $0.11 \mathrm{MPa}$ pressure for $35 \mathrm{~min}$. The explants were cultivated in a thermal room at $24-25^{\circ} \mathrm{C}$ temperature, 65 $70 \%$ relative humidity and 2.5-3 thousand lux light with 16 hours photoperiod. Mathematical processing of the results was carried out using mathematical statistics methods of the Microsoft Office Excel 2007 program.

\section{Discussion}

Selected periods and procedure of step-by-step sterilization allowed obtaining $81 \%$ of sterile explants at injection in the culture in February-March and $90 \%$ of sterile explants of grapes if injection into in vitro culture was carried out in April and early May, during a period of active growth. The signs of growth in form of explication of the first pair of leaves in a non-hormonal medium was revealed for 8-9 day of cultivation. The results of this phase are presented in Table 1. All studied grape varieties had better reaction on addition of cobalt nanoparticles into the nutrient medium, moreover an optimum concentration by growth indices was the medium with $1.0 \mathrm{mg} / \mathrm{l}$ concentration of cobalt nanoparticles (Table 2).

Table 1 Intensity of stratification of explants without hormones in nutrient medium

\begin{tabular}{lllll}
\hline $\begin{array}{l}\text { Period of injection } \\
\text { into culture }\end{array}$ & $\begin{array}{l}\text { Average size of main shoot for the } \\
\text { 30th day of stratification }\end{array}$ & $\begin{array}{l}\text { Number of infected } \\
\text { explants, pcs. }\end{array}$ & $\begin{array}{l}\text { Number of viable } \\
\text { explants, pcs. }\end{array}$ & $\begin{array}{l}\text { Efficiency of } \\
\text { sterilization, \% }\end{array}$ \\
\hline February- March & $4.7 \pm 0.37$ & 6 & 24 & $81.13 \pm 5.1$ \\
April- May & $5.3 \pm 0.39$ & 3 & 27 & $90.00 \pm 3.3$ \\
\hline
\end{tabular}


Table 2 Biometrical indices of grape in nutrient medium for multiplication (day 30 )

\begin{tabular}{llll}
\hline Variant of nutrient medium & Height of shoot, cm & Number of shoots, pcs. & Number of nods, pcs. \\
\hline Control & $4.68 \pm 0.49$ & $1.53 \pm 0.82$ & $5.27 \pm 0.94$ \\
MS Co $(0.5 \mathrm{mg} / \mathrm{l})$ & $4.78 \pm 0.45$ & $1.67 \pm 0.80$ & $5.70 \pm 0.75$ \\
MS Co $(1.0 \mathrm{mg} / \mathrm{l})$ & $5.46 \pm 0.48$ & $2.73 \pm 0.78$ & $7.33 \pm 0.99 *$ \\
MS Co $(1.5 \mathrm{mg} / \mathrm{l})$ & $4.98 \pm 0.44$ & $1.83 \pm 0.75$ & $6.40 \pm 1.30$ \\
MS Cu $(0.5 \mathrm{mg} / \mathrm{l})$ & $4.53 \pm 0.44$ & $1.57 \pm 0.57$ & $5.10 \pm 0.96$ \\
MS Cu (1.0 mg/l) & $4.70 \pm 0.51$ & $1.37 \pm 0.49$ & $4.60 \pm 0.81$ \\
MS Cu (1.5 mg/l) & $4.19 \pm 0.44$ & $1.13 \pm 0.35$ & $3.90 \pm 0.92$ \\
\hline
\end{tabular}

\section{Conclusion}

In conclusion, this method of graft treatment significantly accelerated formation of the size of the main shoot in the graft and the number of formed roots increased. The optimum concentration of copper and cobalt nanoparticles is $1.0 \mathrm{mg} / \mathrm{l}$. Use of copper nanoparticles at $1.0 \mathrm{mg} / 1$ concentration in addition into Murashige and Skoog nutritious medium slightly increases the sprout height by $7.5 \%$ and the number of nodes by $27 \%$. In this case, the multiplication factor is reduced to 1:6. As a result of the study, the optimal cobalt concentration was determined. Addition of the cobalt nanoparticles at a concentration of $1.0 \mathrm{mg} / \mathrm{l}$ into Murashige and Skoog nutritious medium on the clonal micropropagation stage significantly increases the sprout height by $21 \%(\mathrm{a}=0.05)$ and the number of nodes by $70 \%$ $(\mathrm{a}=0.05)$. In this case, the multiplication factor is reduced to $1: 17$.

\section{Author contribution statement}

All the authors participated in the formulation of this experiment. K.V. Liapina participated in the process of obtaining and investigation of the nanoparticles, P.G. Dulnev took part in the process of obtaining and investigation of the growth regulators based on copper and cobalt nanoparticles, E. A. Shkopinskij and T.E. Talankova-Sereda participated in the process of selection of composition of the nutrient media for grapes culture, introduction into in vitro culture, passivation in nutrient media based on nanoparticles, taking biometric indices. All authors reviewed the manuscript.

\section{Acknowledgments}

This work was carried out as a search work.

\section{Conflict of interest}

None.

\section{References}

1. Doroshenko NP, Arestova NO, Sobolev AA. Features of cultivation in vitro of some technical varieties of grapes. Vinodelie I vinogradstvo. 2004;4:34-36.
2. Skorokhod VO. Commercial biotechnology of microclonal reproduction of grapes in culture "in vitro": monograph. Kherson: Ailant; 2000. 327 p.

3. Zlenko VA, Troshin LP, Kotikov IV. Reproduction of grapes by in vitro methods. Part 2: Plant development in vitro and their adaptation to in vivo conditions. Vinograd $i$ vino Rossii. 1998. 5p

4. Doroshenko NP. Production of basic planting material. Vinograd $i$ vino Rossii. 2001;2:23-25.

5. Ivancheva Yu N. Ecological and biological effects of nanopowders of cobalt, copper and copper oxide in plant - animals system: author's abstract. Cand of Biological Sciences. 2012. 21 p.

6. Lopatko KG. Grounds of physical and technical basics of biological functionality metal nanoparticles. Dr Tech Sci, National University of Life and Environmental Sciences of Ukraine, Ukraine, 2015. 46 p.

7. Talankova-Sereda TE, Liapina KV, Shkopinskij EA, et al. The Influence of $\mathrm{Cu}$ and $\mathrm{Co}$ Nanoparticles on Growth Characteristics and Biochemical Structure of Mentha Longifolia in Vitro. Nanosci Nanoeng. 2016;4(2):31-39.

8. Shkopinskij EA, Liapina KV, Talankova-Sereda TE, et al. The influence of $\mathrm{Cu}$ and $\mathrm{Co}$ nanoparticles on Mentha piperita growth and rootage in vitro. Nanophysics, nanophotonics, Surface Studies, and Applications. Springer Physics series; 2016.

9. Ustinov Al, Melnichenko TV, Liapina KV, et al. Patent of Ukraine \#82448 Method to produce encapsulated nanopowders and unit for its implementation. 2008.

10. Ustinov Al, Melnichenko TV, Liapina KV, et al. US Patent \#8491972B2 Method of producing encapsulated nanopowders and installation for its implement. 2013.

11. Dulnev PG, Liapina KV, Davidova OE, et al. Patent of Ukraine \#91374 Sposob dispergirovaniya I stabilizacii nanochastic medi v vodnih sredah. 2014.

12. Kushnir PG, Sarnatska VV. Microcolonal reproduction of plants: theory and practice. Nauk Dumka. 2005. 269 p. 